\title{
Associations of systolic and diastolic blood pressure night-to-day ratios with atherosclerotic cardiovascular diseases
}

\begin{abstract}
Anping Cai ${ }^{1}$, Qi Zhong ${ }^{1}$, Chaofan Liu, Dan Zhou, Xida Li, Ying Zhang, Yingqing Feng and Yingling Zhou
Our objective was to evaluate the associations of the systolic and diastolic blood pressure night-to-day ratios (SBP-NDR and DBP-NDR) with composite atherosclerotic cardiovascular diseases (ASCVDs) comprising coronary heart disease (CHD) and ischemic stroke (IS) cases, respectively. The clinical conditions associated with SBP-NDR and DBP-NDR were also evaluated. A total of 401 patients who underwent 24-h ambulatory BP monitoring were enrolled. In general, the mean age was $59.7 \pm 14.7$ years and male subjects accounted for $59.1 \%$ of the study subjects. Regarding the ASCVD risk factors, $17.0 \%$ of the study subjects smoked, $5.2 \%$ abused alcohol, $2.0 \%$ had a family history of ASCVD, $23.3 \%$ had diabetes and $96.0 \%$ had dyslipidemia. Fifty (12.5\%) and $128(31.9 \%)$ study subjects had a previous diagnosis of CHD and IS, respectively. Dipper and non-dipper pattern-specific differences in clinical characteristics between the SBP-NDR and DBP-NDR categories were observed. The multiple linear regression analysis showed that advanced age, smoking, CHD and IS were positively associated with SBPNDR and statins were inversely associated with SBP-NDR; only IS was positively associated with DBP-NDR. The logistic regression analysis showed that after adjusting for the covariates of age, smoking, alcohol abuse, diabetes, hypertension, dyslipidemia, and SBP and DBP at admission, only DBP-NDR remained significantly associated with composite ASCVD, with an odds ratio of 1.029 (95\% confidence interval 1.002-1.056, $P=0.038$ ). There were significant differences in the associations of SBP-NDR and DBP-NDR with composite ASCVD. Clinical conditions independently associated with SBP-NDR and DBP-NDR were also somewhat different. In a specific population group, DBP-NDR may be superior to SBP-NDR with respect to screening for ASCVD.

Hypertension Research (2016) 39, 874-878; doi:10.1038/hr.2016.82; published online 7 July 2016
\end{abstract}

Keywords: ambulatory blood pressure monitoring; atherosclerotic cardiovascular diseases; dipper; non-dipper

\section{INTRODUCTION}

Hypertension has become a major public health problem in China, owing to its significant contribution to the increasing incidence and prevalence of atherosclerotic cardiovascular diseases (ASCVDs), such as coronary heart disease (CHD) and ischemic stroke (IS), in recent decades. ${ }^{1-3}$ It is therefore clinically important to screen and identify hypertensive subjects who are at high cardiovascular risk, to apply effective and timely preventions that will reduce ASCVD incidence.

Clinic blood pressure (BP) has been shown to be associated with cardiovascular outcomes ${ }^{4,5}$ and it has been used commonly in clinical practice to diagnose hypertension and to evaluate hypertension severity. However, there are several drawbacks of clinic BP measurement, such as a limited number of BP readings and the inability to assess BP variability over a period of time. Compared with clinic BP measurement, 24-h ambulatory BP monitoring (ABPM) has multiple advantages, including that it allows the assessment of hypertension severity, BP variability and therapeutic efficacy for over $24 \mathrm{~h} .{ }^{6}$ Moreover, it is reported that $\mathrm{ABPM}$ is superior to clinic BP in predicting target organ damage and cardiovascular outcomes, ${ }^{7,8}$ and these benefits are attributed to its capacity to assess nighttime BP levels and discriminate BP patterns in terms of dippers and non-dippers. ${ }^{8,9}$

In brief, a nocturnal BP decline is a normal physiological regulation called the dipper BP pattern and blunted decline or rise in nocturnal $\mathrm{BP}$ is termed the non-dipper pattern, which has been revealed to be significantly associated with adverse cardiovascular outcomes. ${ }^{10,11}$ However, whether there are differences in the associations of blunted systolic and diastolic BP (SBP and DBP) decline with ASCVD and whether there are differences in the clinical conditions associated with blunted SBP and DBP decline have not been sufficiently investigated. Moreover, such investigations in Chinese populations are limited. As

Department of Cardiology, Guangdong Cardiovascular Institute, Guangdong Provincial Key Laboratory of Coronary Heart Disease Prevention, Guangdong General Hospital, Guangdong Academy of Medical Sciences, Guangzhou, China

${ }^{1}$ Co-first authors.

Correspondence: Y Feng or Y Zhou, Department of Cardiology, Guangdong Cardiovascular Institute, Guangdong Provincial Key Laboratory of Coronary Heart Disease Prevention, Guangdong General Hospital, Guangdong Academy of Medical Sciences, 106 Zhongshan Road 2, Guangzhou 510080, China.

E-mail: fyq1819@163.com or drcacocolacai@gmail.com

Received 4 April 2016; revised 17 May 2016; accepted 19 May 2016; published online 7 July 2016 
24-h ABPM is increasingly applied and BP patterns are predictors of cardiovascular outcomes, we therefore conducted a cross-sectional study on 401 patients who were referred for 24-h ABPM in the inpatient department of the Guangdong General Hospital, to address the two issues listed above.

\section{SUBJECTS AND METHODS}

\section{Study subjects}

A total of 401 patients who had undergone a 24-h ABPM in our inpatient department were enrolled after oral informed consent was obtained. This study was approved by the Research Ethics Committee of the Guangdong General Hospital. Patients were enrolled from 1 January 2015 to 31 December 2015. In brief, there were two inclusion criteria: study subjects underwent a 24-h ABPM and the data of interest were available; those who did not wish to take part in the present study were excluded. ABPMs were performed on the study subjects, primarily because there was a need to evaluate their 24-h BP variability while they were treated with anti-hypertensive drug therapy; a minority of the study subjects were suspected of hypertension and underwent a 24-h ABPM to reach a definite diagnosis.

\section{BP measurement}

BP measurement at admission was performed after the patients sat quietly for 10 min by using the Omron HEM-7051 device (Omron HealthCare, Kyoto, Japan) and heart rate at admission was simultaneously recorded. For the 24-h ambulatory BP measurement, we used a portable BP monitoring device (model 90207, Space-Labs Medical Inc., Redmond, WA, USA). Ambulatory BP was recorded from the non-dominant arm for $24 \mathrm{~h}$ at 20 -min intervals during the day $(0600-2200 \mathrm{~h})$ and at 30-min intervals during the night $(2200-0600 \mathrm{~h})$. Continuous SBP and DBP night-to-day ratios (SBP-NDR and DBP-NDR) were calculated by dividing the nighttime SBP and DBP values by the daytime SBP and DBP values, respectively. In brief, subjects with SBP-NDR or DBP-NDR $\leqslant 0.90$ were defined as having a dipper BP pattern and $>0.90$ had a non-dipper BP pattern. ${ }^{6}$

\section{Clinical and laboratory data collection}

The clinical data, including demographics (such as age, gender, smoking status, alcohol abuse and family history of ASCVD), previous medical history (including hypertension, diabetes, dyslipidemia, chronic kidney disease, CHD and IS) and present medication use, were recorded on a case report form. Diagnosis of hypertension, diabetes or dyslipidemia was based on self-report or the use of corresponding medications. Diagnosis of CHD was based on clinical symptoms combined with a coronary computed tomography angiography or coronary artery angiography, and diagnosis of IS was based on a focal neurological deficit combined with a computed tomography scan. Fasting venous blood was sampled for measurements of creatinine, fasting plasma glucose, glycated hemoglobin (HbAlc) and the lipid profile.

\section{Statistical analysis}

Standard descriptive statistics were applied in the analysis. Continuous variables were described using the mean and s.d. and categorical variables were described by the number and percentage of cases. The differences were analyzed using the independent $T$-test for continuous variables and $\chi^{2}$-test or Fisher's exact test for categorical variables. The associations of SBP-NDP and DBP-NDR with clinical conditions were assessed by multiple linear regression analyses. Logistic regression analyses were used to evaluate the associations of SBP-NDR and DBP-NDR with composite ASCVD, including CHD and IS. All of the statistical analyses were computed using SPSS 18.0 (SPSS Inc., Chicago, IL, USA). All of the statistical tests were two-sided and considered statistically significant when the $P$-value was $<0.05$.

\section{RESULTS}

\section{General characteristics of study subjects}

Among the 401 study subjects, $263(65.5 \%)$ had a previous diagnosis of hypertension. The mean age was $59.7 \pm 14.7$ years and male subjects accounted for $59.1 \%(n=237)$. SBP and DBP at admission were
$139.1 \pm 20.6$ and $82.4 \pm 15.0 \mathrm{~mm} \mathrm{Hg}$, respectively, and heart rate at admission was $76.9 \pm 12.5$ beats per minute. The mean 24 -h SBP and DBP were $132.7 \pm 20.2$ and $79.7 \pm 12.1 \mathrm{~mm} \mathrm{Hg}$, respectively, and the mean 24 -h heart rate was $72.2 \pm 10.2$ beats per minute. Regarding ASCVD risk factors, $17.0 \%$ of the study subjects smoked, $5.2 \%$ abused alcohol, 2.0\% had a family history of ASCVD, 23.3\% had diabetes and $96.0 \%$ had dyslipidemia. Fifty (12.5\%) and 128 (31.9\%) study subjects had a previous diagnosis of CHD and IS, respectively, and $13(3.2 \%)$ had a diagnosis of chronic kidney disease.

\section{Pattern-specific differences in clinical characteristics by SBP-NDR and DBP-NDR categories}

Based on SBP-NDR, 74.8\% of the 401 study subjects had a non-dipper BP pattern. Compared with subjects who had a dipper BP pattern, those who had a non-dipper BP pattern had higher levels of fasting plasma glucose and blood urine nitrogen. In addition, both mean $24 \mathrm{~h}$ SBP and DBP, and both SBP-NDR $(0.98 \pm 0.06$ vs. $0.86 \pm 0.04)$ and DBP-NDR $(0.97 \pm 0.08$ vs. $0.87 \pm 0.06)$ were also significantly higher in the non-dipper BP pattern group than the dipper BP pattern group $(P<0.05$ for all comparisons). Study subjects with a non-dipper BP pattern were more frequently smokers $(19.7 \% v s .8 .9 \%, P<0.05)$ and had a higher frequency of diabetes $(26.0 \%$ vs. $14.9 \%, P<0.05)$. No significant differences in IS (29.7\% vs. $32.7 \%)$, CHD (7.9\% vs. $14.0 \%)$ and chronic kidney disease $(2.0 \%$ vs. $3.7 \%)$ were observed between subjects with dipper and non-dipper BP patterns.

Based on DBP-NDR, $66.8 \%$ of the 401 study subjects had a non-dipper BP pattern. Compared with subjects who had a dipper BP pattern, those who had a non-dipper BP pattern had higher fasting plasma glucose levels. In addition, SBP at admission, mean $24 \mathrm{~h}$ SBP and both SBP-NDR $(0.98 \pm 0.07$ vs. $0.89 \pm 0.05)$ and DBP-NDR $(0.98 \pm 0.06$ vs. $0.85 \pm 0.05)$ were also significantly higher in the non-dipper BP pattern group than in the dipper BP pattern group $(P<0.05$ for all comparisons). The study subjects with a non-dipper BP pattern had significantly higher frequencies of hypertension (69.0\% vs. $58.6 \%, P<0.05)$ and $\mathrm{CHD}(14.9 \%$ vs. $7.5 \%, P<0.05)$ and IS was marginally higher $(35.1 \%$ vs. $25.6 \%, P=0.054)$. (See Table 1).

\section{Clinical conditions associated with SBP-NDR and DBP-NDR}

Both SBP-NDR and DBP-NDR were analyzed as continuous variables and a multiple linear regression analysis was performed. As shown in Table 2, we observed that advanced age, smoking, CHD and IS were positively associated with SBP-NDR, whereas statins were inversely associated with SBP-NDR. In addition, only IS was positively associated with DBP-NDR.

Associations of SBP-NDR and DBP-NDR with composite ASCVD Because of the relatively small number of $\mathrm{CHD}$ and IS cases in the present study, we defined composite ASCVD as the combination of these cases to decrease the likelihood of logistic model instability when CHD and IS were analyzed separately. In brief, both SBP-NDR and DBP-NDR were analyzed as continuous variables. As presented in Table 3, the logistic regression analysis showed that, in the unadjusted model, both SBP-NDR and DBP-NDR were positively and comparably associated with composite ASCVD. In model 1, after adjustment for age, gender, smoking, alcohol abuse and family history, both SBPNDR and DBP-NDR were still significantly associated with composite ASCVD. In model 2, after further adjustment for diabetes, hypertension and dyslipidemia, the association of SBP-NDR with composite ASCVD was attenuated and nonsignificant, whereas DBP-NDR remained significantly associated with composite ASCVD with an 
Table 1 Pattern-specific differences by SBP-NDR and DBP-NDR categories

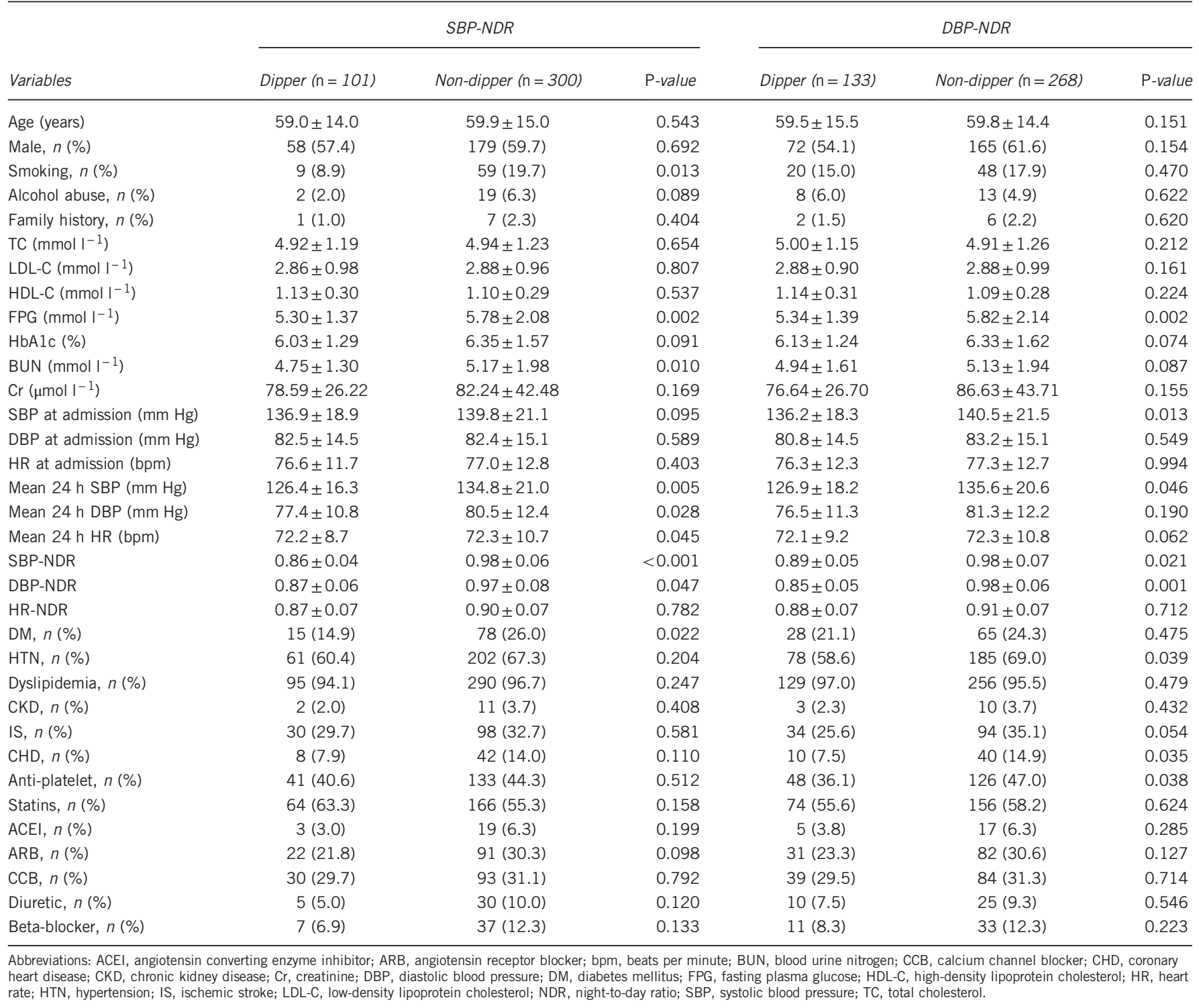

Table 2 Clinical conditions associated with SBP-NDR and DBP-NDR

\begin{tabular}{|c|c|c|c|c|}
\hline Variables & Coefficient $\beta(95 \% \mathrm{Cl})$ & P-value & Coefficient $\beta(95 \%$ Cl) & P-value \\
\hline Male gender & & NS & & NS \\
\hline Smoking & $3.348(0.814-5.882)$ & 0.010 & & NS \\
\hline Alcohol abuse & & NS & & NS \\
\hline DM & & NS & & NS \\
\hline Dyslipidemia & & NS & & NS \\
\hline CKD & & NS & & NS \\
\hline $\mathrm{CHD}$ & $3.364(0.495-6.233)$ & 0.022 & & NS \\
\hline IS & $3.262(1.002-5.522)$ & 0.005 & $2.770(0.263-5.277)$ & 0.030 \\
\hline
\end{tabular}

Abbreviations: Cl, confidence interval; CHD, coronary heart disease; CKD, chronic kidney disease; DBP, diastolic blood pressure; DM, diabetes mellitus; HTN, hypertension; IS, ischemic stroke; NDR, night-to-day ratio; NS, nonsignificant; SBP, systolic blood pressure. 
Table 3 Associations between SBP-NDR and DBP-NDR with composite ASCVD

\begin{tabular}{lcccccc}
\hline & \multicolumn{2}{c}{$S B P-N D R^{*} 100$} & & \multicolumn{2}{c}{$D B P-N D R^{*} 100$} \\
\cline { 2 - 3 } \cline { 5 - 6 } & OR $(95 \% \mathrm{Cl})$ & P-value & & OR $(95 \% \mathrm{Cl})$ & P-value \\
\hline Unadjusted & $1.039(1.011-1.067)$ & 0.006 & & $1.034(1.009-1.060)$ & 0.008 \\
Model 1 & $1.032(1.003-1.062)$ & 0.032 & & $1.027(1.001-1.054)$ & 0.042 \\
Model 2 & & NS & & $1.028(1.001-1.055)$ & 0.043 \\
Model 3 & & NS & & $1.029(1.002-1.056)$ & 0.038 \\
\hline
\end{tabular}

Abbreviations: ASCVD, atherosclerotic cardiovascular disease; $\mathrm{Cl}$, confidence interval;

DBP, diastolic blood pressure; NDR, night-to-day ratio; NS, non-significant; OR, odds ratio; SBP, systolic blood pressure.

odds ratio of 1.028 and $95 \%$ confidence interval of $1.001-1.055$ $(P=0.043)$. In model 3 , after additional adjustment for SBP and DBP levels at admission, the association between DBP-NDR and composite ASCVD was stable with an odds ratio of 1.029 (95\% confidence interval 1.002-1.056, $P=0.038$ ).

\section{DISCUSSION}

The results from our present preliminary study showed that there were substantially significant differences in the associations of SBP-NDR and DBP-NDR with composite ASCVD. In addition, clinical conditions independently associated with SBP-NDR and DBP-NDR were also somewhat different. These findings from Chinese populations are novel and merit further investigation in a prospective and large sample size population study.

Compared with conventional clinic BP measurement, 24-h ambulatory BP measurement has multiple advantages. It can be used to improve diagnostic accuracy by excluding 'white-coat' hypertension and identifying masked hypertension. Moreover, it provides information about BP levels over $24 \mathrm{~h}$ and BP patterns in terms of dippers and non-dippers. In recent decades, a substantial number of epidemiological studies revealed that a 24-h ABPM could provide a more accurate prediction of target organ damage and cardiovascular outcomes. For example, in a population of 1047 untreated Chinese hypertensive patients, Fang-Fei Wei et al. ${ }^{12}$ reported that the 24-h SBP level predicted target organ damage more accurately than conventional BP measurement. Staessen et al. ${ }^{7}$ also recruited untreated hypertensive patients as study subjects and the results from their study showed that nighttime SBP more accurately predicted cardiovascular endpoints than daytime SBP; a higher SBP-NDR was also associated with an increased cardiovascular risk. The results from Dolan Eamon et al. ${ }^{8}$ supported the notion that blunted nocturnal BP decline was a potent predictor of cardiovascular outcome, and that the 24-h ABPM offered comprehensive and integrative BP information on the cardiovascular system and thereby refined cardiovascular risk stratification. ${ }^{13}$ The results from our present cross-sectional study confirmed a close association of continuous SBP-NDR and DBP-NDR with cardiovascular outcomes. Moreover, we additionally observed that DBP-NDR seemed to be superior to SBP-NDR in relation to composite ASCVD. It is conceivable that the pattern-specific differences in clinical characteristics between the SBP-NDR and DBP-NDR categories, as listed in Table 1, might partially explain the discrepancies observed between SBP-NDR and DBP-NDR. However, owing to the relatively small number of ASCVD cases, our present finding might not be significant and future, large-sample-size prospective studies are needed to clarify this preliminary evidence.

Owing to the significant pathophysiological effects of blunted nocturnal $\mathrm{BP}$ decline on the cardiovascular system ${ }^{14,15}$, it is relevant to evaluate clinical conditions associated with a non-dipper BP pattern. In a large cohort of Spanish hypertensive patients, Sierra et al. ${ }^{16}$ reported that in both treated and non-treated patients, advanced age, obesity, diabetes mellitus and overt cardiovascular or renal diseases were associated with a blunted nocturnal BP decline. Similar to these previous findings, we also observed that advanced age and overt cardiovascular diseases such as CHD and IS were associated with a blunted nocturnal BP decline. Nonetheless, some differences in the associations of clinical conditions with SBP-NDR and DBP-NDR were also observed, as seen in Table 2. Pattern-specific differences in clinical characteristics between the SBP-NDR and DBP-NDR categories might also partially explain these observations and a future, large-sample-size prospective study could provide a more comprehensive picture about the differences in associations of SBP-NDR and DBP-NDR with clinical conditions.

Several limitations should be taken into account when interpreting our results. First, this was a cross-sectional study and the results from the present study should not be used to draw causal conclusions. Nonetheless, the novel findings from Chinese populations may be useful for hypothesis generation. Second, the relatively small number of study subjects may not allow us to comprehensively compare the pattern-specific differences in clinical characteristics between the SBPNDR and DBP-NDR categories. Our ongoing study, which plans to recruit more populations referred for 24-h ABPM, may help us further clarify these pattern-specific differences. Third, the number of $\mathrm{CHD}$ and IS cases was also relatively small and the multivariable-adjusted odds ratio estimates of SBP-NDR and DBP-NDR might be unstable. Therefore, we combined CHD and IS cases to form a composite ASCVD group, to reduce the likelihood of model instability. Fourth, it is well known that sleep quality and obstructive sleep apnea have a role in BP pattern and thereby affect the associations of SBP-NDR and DBP-NDR with ASCVD $^{17}$. However, we lacked data about sleep quality and obstructive sleep apnea prevalence in our present study and readers should pay attention to this limitation. In future prospective studies, it is necessary and important to evaluate these two major aspects to improve predictive accuracy. Lastly, the present study only recruited Chinese subjects and this was a single-center study; therefore, these findings should not be extrapolated to other populations.

\section{CONCLUSION}

The findings from our preliminary study have implications for daily clinical practice. Compared with the readily available and free clinic BP measurement, our results justify the broad application of 24-h ABPM to improve both diagnostic and predictive accuracies. In a specific population group, DBP-NDR may be superior to SBP-NDR with respect to screening for ASCVD, and both SBP-NDR and DBPNDR may be beneficial for risk stratification refinement. Certain clinical conditions may predispose hypertensive subjects to the development of either SBP-specific non-dipper patterns or DBPspecific non-dipper patterns, and it is clinically relevant to identify these clinical conditions to prevent or reverse non-dipper BP patterns.

\section{CONFLICT OF INTEREST}

The authors declare no conflict of interest.

\section{ACKNOWLEDGEMENTS}

We thank Cheng Wang for her assistance with the statistical analyses of our paper. This work was supported by the Natural Science Foundation of China (number 81470571), the Natural Science Foundation of Guangdong Province (numbers 2015A030313660 and 2016A030313798), the Scientific Technology 
Project of Guangdong Province (numbers 2014B020212008 and 2014A020212552) and the Scientific Technology Project of GuangZhou (number 1563000381).

1 Critchley J, Liu J, Zhao D, Wei W, Capewell S. Explaining the increase in coronary heart disease mortality in beijing between 1984 and 1999. Circulation 2004; 110: 1236-1244.

2 Kearney PM, Whelton M, Reynolds K, Muntner P, Whelton PK, He J. Global burden of hypertension: analysis of worldwide data. Lancet 2005; 365: 217-223.

3 Murphy SJ, Coughlan CA, Tobin O, Kinsella J, Lonergan R, Gutkin M, McCabe DJ. Continuation and adherence rates on initially-prescribed intensive secondary prevention therapy after rapid access stroke prevention (rasp) service assessment. J Neurol Sci 2016; 361: 13-18.

4 Lewington S, Clarke R, Qizilbash N, Peto R, Collins R, Prospective Studies C. Agespecific relevance of usual blood pressure to vascular mortality: a meta-analysis of individual data for one million adults in 61 prospective studies. Lancet 2002; 360: 1903-1913.

5 Turnbull F, Blood Pressure Lowering Treatment Trialists C. Effects of different bloodpressure-lowering regimens on major cardiovascular events: results of prospectivelydesigned overviews of randomised trials. Lancet 2003; 362: 1527-1535.

6 Parati G, Stergiou G, O'Brien E, Asmar R, Beilin L, Bilo G, Clement D, de la Sierra A, de Leeuw P, Dolan E, Fagard R, Graves J, Head GA, Imai Y, Kario K, Lurbe E, Mallion JM, Mancia G, Mengden T, Myers M, Ogedegbe G, Ohkubo T, Omboni S, Palatini P, Redon J, Ruilope LM, Shennan A, Staessen JA, vanMontfrans G, Verdecchia P, Waeber B, Wang J, Zanchetti A, Zhang Y, European Society of Hypertension Working Group on Blood Pressure M, Cardiovascular V. European society of hypertension practice guidelines for ambulatory blood pressure monitoring. J Hypertens 2014; 32: 1359-1366.

7 Staessen JA, Thijs L, Fagard R, O'Brien ET, Clement D, de Leeuw PW, Mancia G Nachev C, Palatini P, Parati G, Tuomilehto J, Webster J, Investigator SHET. Predicting cardiovascular risk using conventional vs ambulatory blood pressure in older patients with systolic hypertension. J Am Med Assoc 1999; 282: 539-546.

8 Dolan E, Stanton A, Thijs L, Hinedi K, Atkins N, McClory S, Den Hond E, McCormack P Staessen JA, O'Brien E. Superiority of ambulatory over clinic blood pressure measurement in predicting mortality: the Dublin Outcome Study. Hypertension 2005; 46: 156-161.

9 Boggia J, Li Y, Thijs L, Hansen TW, Kikuya M, Bjorklund-Bodegard K, Richart T, Ohkubo T, Kuznetsova T, Torp-Pedersen C, Lind L, Ibsen H, Imai Y, Wang J, Sandoya E, O'Brien E, Staessen JA, International Database on Ambulatory Blood Pressure Monitoring in Relation to Cardiovascular Outcomes (IDACO) Investigatots. Prognostic accuracy of day versus night ambulatory blood pressure: a cohort study. Lancet 2007; 370: 1219-1229.

10 Ohkubo T, Hozawa A, Yamaguchi J, Kikuya M, Ohmori K, Michimata M, Matsubara M, Hashimoto J, Hoshi H, Araki T, Tsuji I, Satoh H, Hisamichi S, Imai Y. Prognostic significance of the nocturnal decline in blood pressure in individuals with and without high 24-h blood pressure: the Ohasama Study. J Hypertens 2002; 20: 2183-2189.

11 Fagard RH, Celis H, Thijs L, Staessen JA, Clement DL, De Buyzere ML, De Bacquer DA. Daytime and nighttime blood pressure as predictors of death and cause-specific cardiovascular events in hypertension. Hypertension 2008; 51: 55-61.

12 Wei FF, Li Y, Zhang L, Xu TY, Ding FH, Staessen JA, Wang JG. Association of target organ damage with 24-hour systolic and diastolic blood pressure levels and hypertension subtypes in untreated chinese. Hypertension 2014; 63: 222-228.

13 Clement DL, De Buyzere ML, De Bacquer DA, de Leeuw PW, Duprez DA, Fagard RH, Gheeraert PJ, Missault LH, Braun JJ, Six RO, Van Der Niepen P, O'Brien E, Office versus Ambulatory Pressure Study Investigators. Prognostic value of ambulatory bloodpressure recordings in patients with treated hypertension. N Engl J Med 2003; 348: 2407-2415.

14 Yano Y, Kario K. Nocturnal blood pressure and cardiovascular disease: a review of recent advances. Hypertens Res 2012; 35: 695-701.

15 Celik G, Yilmaz S, Ergulu Esmen S. Non-dipping blood pressure patterns and arterial stiffness parameters in patients with behcet's disease. Hypertens Res 2015; 38 : $856-861$.

16 de la Sierra A, Redon J, Banegas JR, Segura J, Parati G, Gorostidi M, de la Cruz JJ, Sobrino J, Llisterri JL, Alonso J, Vinyoles E, Pallares V, Sarria A, Aranda P, Ruilope LM, Spanish Society of Hypertension Ambulatory Blood Pressure Monitoring Registry Investigators. Prevalence and factors associated with circadian blood pressure patterns in hypertensive patients. Hypertension 2009; 53: 466-472.

17 Cai A, Wang L, Zhou Y. Hypertension and obstructive sleep apnea. Hypertens Res 2016; 39: 391-395. 